\title{
Super Vertex Graceful Graphs
}

\author{
N. Murugesan \\ Post graduate Department of Mathematics, \\ Government Arts College (Autonomous), \\ Coimbatore- India
}

\author{
R.Uma \\ Department of Mathematics \\ Sree Saraswathi Thyagaraja College \\ (Autonomous), Pollachi, Coimbatore, India.
}

\begin{abstract}
For a defined graph labeling, there exists a number of bijective functions for a graph of defined order and size which leads to different graphs. In this paper, a mathematical tool is developed to find the number of super vertex graceful graphs for a defined order " $p$ " and size " $q$ ".
\end{abstract}

\section{Mathematics Subject Classification:} 05C78-

\section{Keywords}

Order of a graph, size of a graph, graceful graphs, super vertex graceful graphs.

\section{INTRODUCTION}

In this paper, graph means a simple undirected graph. Labeling of a graph is the process of assigning values to vertices or edges or both subject to certain conditions. This concept of labelling was introduces by A. Rosa in the name of $\beta$ - valuation in $1960 \mathrm{~s}$. Later. It was named as graceful labelling by Golomb. Since then many types of labelling came into existence. A detailed report on the labelling of graphs is given by Gallian [1]. In 2009, Sin Min Lee and others [9] defined super vertex graceful labelling and analysed the super vertex gracefulness of unicyclic graphs under this labelling. N. Murugesan, R. Uma $[4,5,6]$ have analysed complete bipartite graphs, twigs, spiders, regular caterpillars, fire cracker graphs under the same labeling. and some properties of super vertex graceful graphs. In this paper, a model is defined to find the number of super vertex graceful graphs for defined order "p" and size "q".

\section{DEFINITION}

\subsection{Super vertex graceful labelling:}

A graph $\mathrm{G}$ with $p$ vertices and $q$ edges, vertex set $\mathrm{V}(\mathrm{G})$ and edge set $\mathrm{E}(\mathrm{G})$, is said to be super vertex graceful ( $S V G$ ), if there exists a function pair $\left(f, f^{+}\right)$where $\mathrm{f}$ is a bijection from $\mathrm{V}(\mathrm{G})$ onto $\mathrm{P}, \mathrm{f}^{+}$is a bijection from $\mathrm{E}(\mathrm{G})$ onto $\mathrm{Q}$, such that $f^{+}(u, v)=f(u)+f(v) \quad$ for any $(u, v) \in$ $V(G)$, where $\mathrm{P}$ and $\mathrm{Q}$ are finite set of integers defined and as follows:

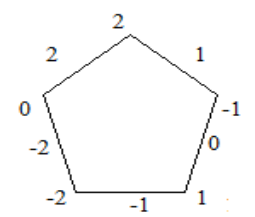

Figure 1 Super vertex graceful graph $\mathbf{C}_{5}$
$P=\left\{\begin{array}{c} \pm 1, \pm 2, \ldots, \pm \frac{p}{2} \text { if } p \text { is even } \\ 0, \pm 1, \pm 2, \ldots, \pm \frac{p_{-}-1}{2} \text { if } p \text { is odd. }\end{array} \quad Q=\right.$

$\left\{\begin{array}{c} \pm 1, \pm 2, \ldots, \pm \frac{p}{2} \text { if } q \text { is even } \\ 0, \pm 1, \pm 2, \ldots, \pm \frac{p-1}{2} \text { if } q \text { is odd. }\end{array}\right.$

\subsection{Example}

In the graph given in fig 2 , both the size and order is 5 . Therefore $\mathrm{P}=\mathrm{Q}=\{-2,-1,0,1,2\}$. Here $\mathrm{f}^{+}$and $\mathrm{f}$ are defined such that $\mathbf{f}^{+}(-1,1)=0 ; \mathbf{f}^{+}(-1,2)=1 ; \mathbf{f}^{+}(0,2)=2 ; \mathbf{f}^{+}(1,-2)=-1 ; \mathbf{f}^{+}(-$ $2,0)=-2$.Then $G$ is SVG. It is interesting to note that $C_{5}$ is SVG. But Golomb [2], discussed that $\mathrm{C}_{5}$ is not graceful.

\section{RESULT}

In labeling, many types of graphs exist for a defined order and size. Similarly, for a particular graph, there exist different types of graphs with same type of labeling. In this section this concept is explained in the context of super vertex graceful labeling.

\subsection{Theorem}

Let $G$ be a graph of order ' $p$ ' and size ' $q$ '. Then by the definition of super vertex graceful graphs the vertex set label ' $\mathrm{P}$ ' and edge set label ' $\mathrm{Q}$ ' are

$$
\begin{aligned}
& P=\left\{\begin{array}{l} 
\pm 1, \pm 2, \ldots \pm \frac{p}{2} \text { ifp is even } \\
0, \pm 1, \pm 2, \ldots \pm \frac{p-1}{2} \text { ifpisodd }
\end{array}\right. \\
& Q=\left\{\begin{array}{l} 
\pm 1, \pm 2, \ldots \pm \frac{q}{2} \text { if qiseven } \\
0, \pm 1, \pm 2, \ldots \pm \frac{q-1}{2} \text { if qisodd }
\end{array}\right.
\end{aligned}
$$

The edge set labels are obtained from the vertex labels. The number of ways of obtaining the edge label $+\mathrm{k}$ and $-\mathrm{k}$ are the same. The following set of ordered pairs induces the particular edge label.

The set of ordered pairs depends on the size of the graph (odd or even)

In order to form super vertex graceful graph of order ' $q$ ' (odd) we have to select one order pair from each row of table 1 . The number of ways selection of order pairs of label ' $i$ ' is $\alpha_{i}$ for each label i. Therefore the edge label 0 can be obtained from $\alpha_{0}$ ways. If the order pair selected is $(1,-1)$, for the edge label 0 , the order pair for edge label ' 1 ' can be obtained 
from $\alpha_{1}$ order pairs, similarly, the edge label 2 can obtained from $\alpha_{2}$ pairs etc. So, the number of ways of obtaining the edges $1,2, \ldots \frac{q-1}{2}$ is $\alpha_{1} \cdot \alpha_{2} \ldots . \alpha_{\frac{q-1}{2}}$. Similar argument can be applied for the edge labels $-1,-2, \ldots-\frac{q-1}{2}$. Hence the number of super vertex graceful graphs is 2. $\alpha_{0} \cdot \alpha_{1} \cdot \alpha_{2} \ldots \alpha_{\frac{q-1}{2}}$ if 'q' is odd.

Also, if ' $p$ ' is even $0 \notin P$ and the corresponding number of ordered pairs reduces by ' 1 ' for all the edge labels in the second column of the tables 1 and 2 .

\subsection{Example}

Let us consider a graph of order 6 and size 10. By the definition of super vertex graceful map, vertex label $\mathrm{P}$ and edge label set $\mathrm{Q}$ are defined as $P=\{ \pm 1, \pm 2, \pm 3\}$ and $Q=\{ \pm 1, \pm 2, \pm 3, \pm 4, \pm 5\}$.The ordered pairs that induce the edge labels from the given vertex labels are given in the table 3 . The vertex pairs $(-1,3),(1,2),(1,3),(2,3),(1,-$ $3),(-1,-2),(-1,-3),(-2,-3)$ belong to all the set of ordered pairs given in the second column.

\subsection{Remark}

The super vertex graceful graphs obtained from the above given methodology is connected only if the union of the elements of the ordered pairs is equal to the vertex label set $P$. Otherwise the graph is disconnected.The above concept is illustrated by an example.

Let us consider the graph of order ' 7 ' and size ' 9 '. Then $P=\{ \pm 1, \pm 2, \pm 3\}$ and $\quad Q=$ $\{0, \pm 1, \pm 2, \pm 3, \pm 4\}$. The induced edge labels and the set of ordered pairs are tabulated in table 4 .

Let us consider the following set of ordered pairs which induce the labels $0,1,2,3,4,-1,-2,-3,-4$ be $(1,-1),(0,1),(-$ $1,3),(0,3),(1,3),(0,-1),(0,-2),(0,-3),(-1,-3)$. In the above set of ordered pairs the vertex label 2 is missing. The following graph with this set of vertex label is shown below and it is disconnected.

Table 1 Edge labels and the set of ordered pairs if ' $q$ ' is odd

\begin{tabular}{|c|c|c|}
\hline $\begin{array}{l}\text { Edge } \\
\text { Label }\end{array}$ & Set of ordered pairs & No. of ordered pairs \\
\hline 0 & $(1,-1),(2,-2),(3,-3), \ldots\left(-\left(\frac{q-1}{2}\right),\left(\frac{q-1}{2}\right)\right)$ & $\alpha_{0}$ \\
\hline 1 & $(0,1),(-1,2),(-2,3), \ldots .\left(-\left(\frac{q-3}{2}\right),\left(\frac{q-1}{2}\right)\right)$ & $\alpha_{1}$ \\
\hline 2 & $(0,2),(-1,3),(-2,4), \ldots .\left(-\left(\frac{q-5}{2}\right),\left(\frac{q-1}{2}\right)\right)$ & $\alpha_{2}$ \\
\hline$\cdots$ & $\cdots$ & \\
\hline$\cdots$ & $\cdots$ & \\
\hline$\ldots$ & $\ldots$ & \\
\hline$\frac{q-1}{2}$ & $\left(0,\left(\frac{q-1}{2}\right)\right)$ & $\alpha_{\frac{q-1}{2}}$ \\
\hline-1 & $(0,-1),(1,-2),(2,-3), \ldots .\left(\left(\frac{q-3}{2}\right),-\left(\frac{q-1}{2}\right)\right)$ & $\alpha_{1}$ \\
\hline-2 & $(0,-2),(1,-3),(2,-4), \ldots .\left(\left(\frac{q-5}{2}\right),-\left(\frac{q-1}{2}\right)\right)$ & $\alpha_{2}$ \\
\hline$\cdots$ & $\cdots$ & $\cdots$ \\
\hline$\cdots$ & $\cdots$ & $\cdots$ \\
\hline$-\left(\frac{q-1}{2}\right)$ & $\left(0,-\left(\frac{q-1}{2}\right)\right)$ & $\frac{\alpha_{q-1}}{2}$ \\
\hline
\end{tabular}


Table 2. Edge labels and the set of ordered pairs if ' $q$ ' is even

\begin{tabular}{|c|c|c|}
\hline Edge Label & Set of ordered pairs & No. of ordered pairs \\
\hline 1 & $(0,1),(-1,2),(-2,3), \ldots .\left(-\left(\frac{q-2}{2}\right),\left(\frac{q}{2}\right)\right)$ & $\alpha_{1}$ \\
\hline 2 & $(0,2),(-1,3),(-2,4), \ldots .\left(-\left(\frac{q-4}{2}\right),\left(\frac{q}{2}\right)\right)$ & $\alpha_{2}$ \\
\hline$\ldots$ & $\ldots$ & $\ldots$ \\
\hline$\ldots$ & $\cdots$ & $\ldots$ \\
\hline$\frac{q}{2}$ & $\left(0,\left(\frac{q}{2}\right)\right)$ & $\alpha_{\frac{q}{2}}$ \\
\hline-1 & $(0,-1),(1,-2),(2,-3), \ldots .\left(\left(\frac{q-2}{2}\right),-\left(\frac{q}{2}\right)\right)$ & $\alpha_{1}$ \\
\hline-2 & $\begin{array}{l}(0,-2), \quad(1, \\
\ldots .\left(\left(\frac{q-4}{2}\right),-\left(\frac{q}{2}\right)\right)\end{array}$ & $\alpha_{2}$ \\
\hline$\cdots$ & $\cdots$ & $\cdots$ \\
\hline$\cdots$ & $\ldots$ & $\ldots$ \\
\hline$-\left(\frac{q-1}{2}\right)$ & $\left(0,-\left(\frac{q}{2}\right)\right)$ & $\alpha \frac{q}{2}$ \\
\hline
\end{tabular}

In order to form super vertex graceful graph of order ' $q$ ' (odd) we have to select one order pair from each row of table 1 . The number of ways selection of order pairs of label ' $i$ ' is $\alpha_{i}$ for each label i. Therefore the edge label 0 can be obtained from $\alpha_{0}$ ways. If the order pair selected is $(1,-1)$, for the edge label 0 , the order pair for edge label ' 1 ' can be obtained from $\alpha_{1}$ order pairs, similarly, the edge label 2 can obtained from $\alpha_{2}$ pairs etc. So, the number of ways of obtaining the edges $1,2, \ldots \frac{q-1}{2}$ is $\alpha_{1} \cdot \alpha_{2} \ldots . \alpha_{\frac{q-1}{2}}$. Similar argument can be applied for the edge labels $-1,-2, \ldots-\frac{q-1}{2}$. Hence the number of super vertex graceful graphs is 2. $\alpha_{0} \cdot \alpha_{1} \cdot \alpha_{2} \ldots, \alpha_{\frac{q-1}{2}}$ if 'q' is odd.
Also, if ' $\mathrm{p}$ ' is even $0 \notin P$ and the corresponding number of ordered pairs reduces by ' 1 ' for all the edge labels in the second column of the tables 1 and 2 .

\subsubsection{Example:}

Let us consider a graph of order 6 and size 10. By the definition of super vertex graceful map, vertex label $\mathrm{P}$ and edge label set $\mathrm{Q}$ are defined as $P=\{ \pm 1, \pm 2, \pm 3\}$ and $Q=\{ \pm 1, \pm 2, \pm 3, \pm 4, \pm 5\}$.The ordered pairs that induce the edge labels from the given vertex labels are given in the table 3 .

Table 3. Edge labels and the set of ordered pairs if $q=\mathbf{1 0}$

\begin{tabular}{|c|c|c|}
\hline Edge Label & Set of ordered pairs & No. of ordered pairs \\
\hline 1 & $(-1,2),(-2,3)$ & 2 \\
\hline 2 & $(-1,3)$ & 1 \\
\hline 3 & $(1,2)$ & 1 \\
\hline 4 & $(1,3)$ & 1 \\
\hline 5 & $(2,3)$ & 2 \\
\hline-1 & $(1,-2),(2,-3)$ & \\
\hline
\end{tabular}




\begin{tabular}{|c|c|c|}
\hline-2 & $(1,-3)$ & 1 \\
\hline-3 & $(-1,-2)$ & 1 \\
\hline-4 & $(-1,-3)$ & 1 \\
\hline-5 & $(-2,-3)$ & 1 \\
\hline
\end{tabular}

Table. 4 List of ordered pair inducing required edge labels for $q=\mathbf{1 0}$

\begin{tabular}{|l|l|l|}
\hline \multirow{2}{*}{ Proper choice of labels } & Corresponding ordered pairs that induce the label & \multirow{2}{*}{ No. of ways } \\
\hline \multirow{3}{*}{$(1,2,3,4,5,-1,-2,-3-4,-5)$} & $\begin{array}{l}(-1,2),(-1,3),(1,2),(1,3),(2,3),(1,-2),(1,-3),(-1,- \\
2),(-1,-3),(-2,-3)\end{array}$ & \multirow{2}{*}{2} \\
\cline { 2 - 3 } & $\begin{array}{l}(-2,3),(-1,3),(1,2),(1,3),(2,3)(1,-2),(1,-3),(-1,-2), \\
(-1,-3),(-2,-3)\end{array}$ & \\
\cline { 1 - 2 }$(1,2,3,4,5,-1,-2,-3-4,-5)$ & $\begin{array}{l}(-1,2),(-1,3),(1,2),(1,3),(2,3)(2,-3),(1,-3),(-1,- \\
2),(-1,-3),(-2,-3)\end{array}$ & \\
\hline & $\begin{array}{l}(-2,3),(-1,3),(1,2),(1,3),(2,3)(2,-3),(1,-3),(-1,-2), \\
(-1,-3),(-2,-3)\end{array}$ & \\
\hline & & \\
\hline
\end{tabular}
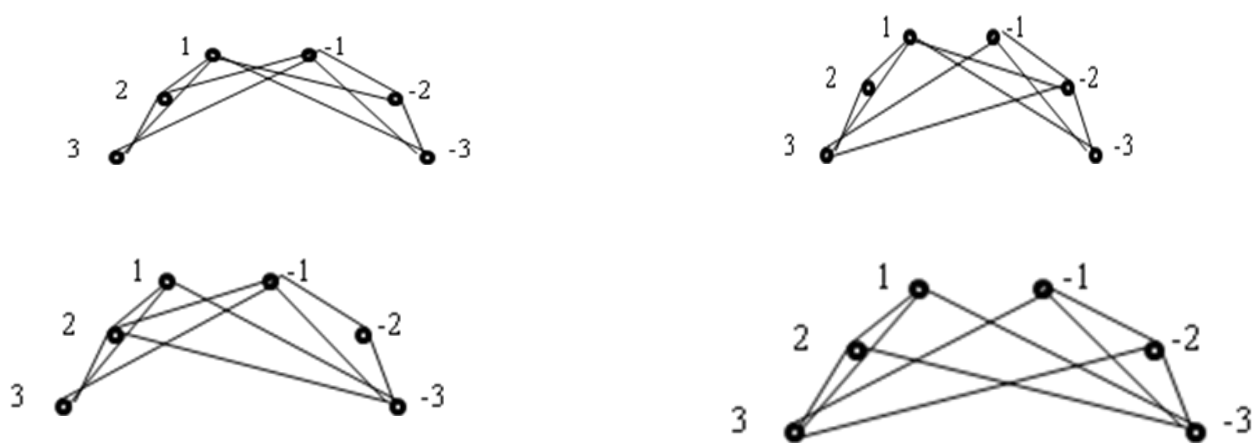

Figure 2 Super vertex graceful graphs of order 6 and size 10

The vertex pairs $(-1,3),(1,2),(1,3),(2,3),(1,-3),(-1,-2),(-1$, $-3),(-2,-3)$ belong to all the set of ordered pairs given in the second column.

\subsubsection{Remark}

The super vertex graceful graphs obtained from the above given methodology is connected only if the union of the elements of the ordered pairs is equal to the vertex label set $\mathrm{P}$.
Otherwise the graph is disconnected.The above concept is illustrated by an example.

Let us consider the graph of order ' 7 ' and size ' 9 '. Then $P=\{ \pm 1, \pm 2, \pm 3\}$ and $\quad Q=$ $\{0, \pm 1, \pm 2, \pm 3, \pm 4\}$. The induced edge labels and the set of ordered pairs are tabulated in table 5 .

Table 5. Edge labels and the set of ordered pairs if $q=9$

\begin{tabular}{|l|c|c|}
\hline Edge Label & Set of ordered pairs & $\begin{array}{l}\text { Number of ordered } \\
\text { pairs }\end{array}$ \\
\hline 0 & $(1,-1),(2,-2),(3,-3)$ & 3 \\
\hline 1 & $(0,1),(-1,2),(-2,3)$ & 1 \\
\hline 2 & $(0,2),(-1,3)$ & 3 \\
\hline
\end{tabular}




\begin{tabular}{|l|c|c|}
\hline 3 & $(0,3),(1,2)$ & 1 \\
\hline 4 & $(1,3)$ & 1 \\
\hline-1 & $(0,-1),(1,-2),(2,-3)$ & 1 \\
\hline-2 & $(0,-2),(1,-3)$ & 1 \\
\hline-3 & $(0,-3),(-1,-2)$ & 1 \\
\hline-4 & $(-1,-3)$ & \\
\hline
\end{tabular}

Let us consider the following set of ordered pairs which induce the labels $0,1,2,3,4,-1,-2,-3,-4$ be $(1,-1),(0,1)$, ($1,3),(0,3),(1,3),(0,-1),(0,-2),(0,-3),(-1,-3)$. In the above set of ordered pairs the vertex label 2 is missing. The following graph with this set of vertex label is shown below and it is disconnected.

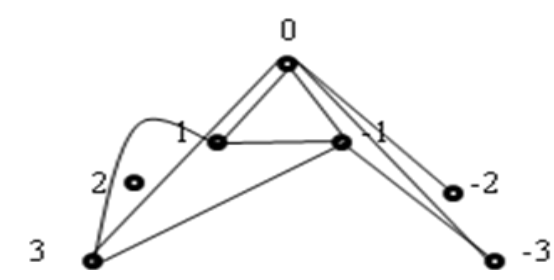

Figure 3. Disconnected super vertex graceful graph

\section{ACKNOWLEDGMENTS}

The project is supported by UGC under the minor research project scheme 2014-2015.

\section{REFERENCES}

[1] Joseph A. Gallian, A Dynamic survey of Graph Labeling, 2008.

[2] Murugesan. N, Uma. R, A Conjecture on Amalgamation of graceful graphs with star graphs, Int.J.Contemp.Math.Sciences, Vol.7, 2012, No.39, 19091919.

[3] Murugesan. N, Uma.R, Super vertex gracefulness of complete bipartite graphs, International J.of Math.Sci \& Engg. Appls, Vol.5, No.VI (Nov, 2011), PP 215-221.

[4] Murugesan. N, Uma. R, Graceful labeling of some graphs and their subgraphs, Asian Journal of Current Engineering and Maths1:6 Nov - Dec (2012) $367-370$.

[5] Murugesan. N, Uma.R Fibonacci gracefulness of $P_{n}{ }_{n}^{2}$ and PP @SQ , International J. of Math. Sci. \& Engg. Appls, , Vol. 7 No. IV (July, 2013), pp. 429-437
[6] N.Murugesan, R.Uma A Study on Super Vertex Graceful Graphs International Journal of Computer Applications (0975 - 8887) Vol 95- No. 10, June 2014

[7] N.Murugesan, R.Uma Super vertex Gracefulness of Some Special Graphs IOSR Journal of Mathematics (IOSRJM) 2319-765X. Volume 11, Issue 3 Vol. V (May - Jun. 2015), PP 07-15

[8] Harary, Graph Theory, Narosa Publishing House, 2001.

[9] A Rosa, On certain valuations of the vertices of a graph, theory Of Graphs (Internet. Sympos., Rome, 1996), Gordon and Breach, Newyork, 1967, pp. 349-355.

[10] Sin - Min - Lee, Elo Leung and Ho Kuen Ng, On Super vertex graceful unicyclic graphs, Czechoslovak mathematical Journal, 59 (134) (2009), 1- 22.

[11] Solairaju. A, Vimala. C, Sasikala. A, Edge - Odd gracefulness of $\mathrm{P}_{\mathrm{M}} \Theta \mathrm{S}_{\mathrm{N}}$, for $\mathrm{M}=5,6,7,8$, International Journal of Computer applications (0975 - 8887), Volume 9- No. 12, November 2010. 